\title{
Análisis de la calidad del servicio y la satisfacción del cliente en la práctica de rafting en el cantón Tena
}

\author{
(c) (1) (1) (1) \\ Analysis of the quality of service and customer satisfaction in the practice \\ of rafting in the Tena canton
}

\author{
Álvaro Santiago Toalombo Díaz. ${ }^{1}$, Tania Cristina Cevallos Punguil. ${ }^{2}$, Gary Patricio \\ Rivadeneyra Olalla. ${ }^{3} \&$ Esteban Alejandro Aguilar Soria. ${ }^{4}$
}

Recibido: 11-04-2021 / Revisado: 19-04-2021 /Aceptado: 12-05-2021/ Publicado: 05-06-2021

\begin{abstract}
.
DOI: https://doi.org/10.33262/concienciadigital.v4i2.2.1784

Rafting is an activity that consists of navigating rivers, controlled by the navigator using oars, with equipment and trained personnel to guide the route. The purpose of this research is to analyze the quality of service and customer satisfaction in the practice of rafting in the Tena canton, year 2020. The design of the research was quantitative and descriptive, with the use of statistical methods of correlational coefficient and one-way ANOVA analysis of variance. A SERVQUAL model survey was applied to a sample of 385 clients of the travel agencies of the Tena canton. Among the results, it is evident that the quality index reflects tangibility as the dimension with the lowest average perception, the calculation of the General average of the 5 dimensions valued over a value of 4.06 represents $81.44 \%$ of the clients who perceive a good quality service according to the percentage degree $0-100 \%$ of the Likert scale. It was concluded that the highest gaps with the perception of the client and their expectation are tangibility (-0.06), reliability (-0.06) and empathy (-0.05), points where it is necessary to implement quality strategies, the Hypothesis analysis reflects a correlation coefficient on service quality and customer

\footnotetext{
1 Universidad Estatal Amazónica, Centro de Posgrado, Maestrante en Turismo, Mención Gestión del Turismo, Puyo-Ecuador, as.toalombod@uea.edu.ec

2 Universidad Estatal Amazónica, Facultad Ciencias de la Vida, Carrera de Turismo, Puyo-Ecuador, tc.cevallosp@uea.edu.ec

${ }^{3}$ Instituto Superior Tecnológico Tena, Docente de la carrera de Gestión de operaciones turísticas, TenaEcuador, spachamama@hotmail.com

${ }^{4}$ Instituto de Formación en Gastronomía Turismo y Hotelería, Docente de Turismo, Guianza, Patrimonio Cultural y Arte Universal Ecuatoriana, Baños-Ecuador, esteban89128@ hotmail.com
} 
satisfaction with 0.828 and a significant one of 0.01 , multivariate correlation analysis of quality dimensions such as tangibility and responsiveness, no are related to the customer's perception of the service.

Keywords: Adventure, extreme sport, Servqual, tourism

\section{Resumen}

El rafting es una actividad que consiste en navegar ríos, controlada por el navegante empleando remos, con un equipamiento y personal capacitado para la guianza en el recorrido. El propósito de esta investigación es analizar la calidad del servicio y satisfacción del cliente en la práctica del rafting en el cantón Tena, año 2020. El diseño de la investigación fue cuantitativo y de tipo descriptivo, con el uso de métodos estadísticos de coeficiente correlacional y análisis de varianza ANOVA unifactorial. Se aplicó una encuesta de modelo SERVQUAL, a una muestra de 385 clientes de las Agencias de viaje del cantón Tena, Entre los resultados se evidencia que el índice de la calidad refleja a la tangibilidad como la dimensión con menor promedio de percepción, el cálculo del promedio general de las 5 dimensiones valoradas sobre un valor de 4.06 representa $81.44 \%$ de los clientes que perciben un buen servicio de calidad de acuerdo al grado de porcentaje 0-100\% de la escala de Likert. Se concluyó que las brechas más altas con la percepción del cliente y su expectativa son tangibilidad $(-0,06)$, fiabilidad $(-0,06)$ y empatía $(-0,05)$, puntos donde se necesita implementar estrategias de calidad, el análisis de las hipótesis, refleja un coeficiente de correlación sobre la calidad del servicio y la satisfacción del cliente con un 0,828 y una significativa de 0,01, el análisis de la correlación multivariente las dimensiones del calidad tales como tangibilidad y capacidad de respuesta, no se relacionan con la percepción del cliente frente al servicio.

Palabras clave: Aventura, deporte extremo, Servqual, turismo

\section{Introducción.}

Actualmente las organizaciones de servicios se ven en la necesidad de brindar mejor calidad y satisfacción al cliente, no solo es la competencia que exige un mayor esmero en el servicio ya que esto está enfocado al también al prestigio de la marca y al nivel de capacitación de recepción a los clientes; es importante que las organizaciones desarrollen planes de mejora continua mediante lo cual se efectúe un nivel de excelencia en los servicios ofertados de tal forma que se puedan satisfacer en gran medida al cliente.

La operación turística de aventura para la prestación del servicio en Tena dinamiza la economía local aprovechando los recursos naturales y a la vez culturales de la localidad y puede convertirse en una fuente inagotable de alternativas para el desarrollo de servicios que promuevan el rescate de identidad y protección de los escenarios naturales. Con el fin de brindar calidad en el servicio apoyándose en normas y reglamentos que permitan impulsar el potencial turístico en el lugar con actividades de aventura. 
Partiendo de un concepto de potencial turístico como afirma García, (2012), le reconoce a la "aptitud para el desarrollo turístico de un lugar donde existen atractivos turísticos disponibles en cantidad y calidad, explotables para el óptimo desarrollo de la actividad misma" (pág. 8).

Mientras los autores Cámara y Ángeles, (2014) refieren que es "cualquier elemento, material o inmaterial, vinculado a la naturaleza o a la cultura de una localidad, que no se encuentran todavía incorporados a la dinámica turística por el hombre, ni cuenta con ningún tipo de servicios de apoyo a fines turísticos" (pág. 35).

Otro criterio es planteado por la autora Mikery J. (2014), al comentar que el potencial turístico está comprendido como aquellas características físico-geográficas, con determinada infraestructura y sociedad que favorecen a una región atraer turistas.

Con estas primicias es importante definir que el potencial turístico y la satisfacción del cliente son parte imprescindible en la toma de decisiones de los clientes para promover un lugar y generar servicios de calidad.

"En la actualidad, lograr la plena" satisfacción del cliente" es un requisito indispensable para ganarse un lugar en la" mente" de los clientes y, por ende, en el mercado meta" (Thompson, 2005, pág. 1).

Por otra parte, desde la perspectiva del marketing: "la satisfacción de las necesidades de los clientes es la clave de los intercambios entre empresas y mercado, y desde los orígenes del marketing, la satisfacción ha sido considerada como el factor determinante del éxito en los mercados" (Gil, Sánchez, Berenguer, y González- Gallarda, 2005, pág. 53).

Ademas es necesario entender de manera mas amplia el servicio Para entender mejor lo que es un servicio, hay que mencionar a la Real Academia Española, en donde etimológicamente el servicio proviene del latín servitium cuyo significado es 'esclavitud, servidumbre'. Esto debe porque en la antigüedad, el servicio era el término que originalmente se encontraba asociado al trabajo que los sirvientes o siervos quienes realizaban u ofrecían su trabajo a sus amos o feudales que estos últimos imponían. Con el paso del tiempo lo define al servicio como:

Las actividades ofrecidas, por una parte, que constituyen el componente de naturaleza más o menos intangible destinado a satisfacer las necesidades de la otra parte, la cual desea recibir un beneficio a través de tales actividades que se producen como resultado de la interacción de los deseos de las dos partes (Aguiar, 2001).

De tal modo que para medir los índices de satisfacción existen muchos modelos que se pueden aplicar para tener en cuenta cuan eficiente es el servicio que se está brindando, uno de los principales y más usados es el Modelo SERVQUAL (Service Quality) propuesto por Parasuraman, Zeithaml y Berry en el año 1985 el cual posee la finalidad para medir las expectativas al igual que las percepciones del cliente todo ello respecto al servicio brindado que una organización o empresa ofrece al cliente, de manera 
imprescindible se debe considerar diversas dimensiones para fijar y definir la calidad del servicio; a partir de una serie de preguntas estructuradas que ha formulado y diseñado el modelo de las cuales se puede obtener las dimensiones.

Es inherente señalar que el modelo SERVQUAL permite medir 2 etapas o fase decisivas en el servicio: la primera es consistente con la entrega del servicio, es decir, las expectativas sobre él, mientras que la segunda se basa estrictamente luego de consumir el servicio que consiste en la percepción; basado en un rango o escala de cinco puntos concernientes a las dimensiones en conjunto con 22 ítems que se analizan (Saghier, 2013; Al Khattab y Aldehayyat, 2011).

Es necesario incluir su aplicación a los servicios de cualquier tipo Daza, (2013); del mismo modo López, (2018) presenta la siguiente afirmación sobre la escala SERVQUAL la cual da la posibilidad de: "medir y relacionar la percepción del cliente con las expectativas que tiene la empresa respecto a la calidad del servicio o el producto que se produzca, comercialice o distribuya", de tal forma que si el puntaje de las percepciones supera o es mayor a las expectativas el resultado indica que la calidad de servicio es alta de acuerdo con (Parasuraman, et al., 1988).

Además, del modelo SERVQUAL está el modelo SERVPERF (Service Performance) el cual fue propuesto por Cronin y Taylor, S. A. en el año 1992, anterior a una investigación empírica que se sometió a distintas industrias dedicadas al servicio mediante lo cual se llegaron a la conclusión que SERVQUAL el modelo propuesto por autores anteriores no consiste en un modelo adecuado que posibilite medir eficientemente la calidad de servicio; por tal razón, SERVPERF es consistente con un modelo establecido únicamente dentro de las percepciones con el objetivo de eliminar las expectativas como lo comento (Cronin y Taylor, 1992).

Por ende, es evidente que lo anterior mente expuesto por los actores se fundamenta en firmes argumentos respecto al modelo SERVQUAL mostrando que las expectativas no son una especifica definición a perseguir por lo que resulta difícil distinguir en su contexto la variabilidad que abarca esta definición, que por lo tanto el modelo SERVPERF es utilizado como una herramienta que facilita examinar el desempeño dentro de un establecimiento y su servicio el cual arroja resultados de una medición en la efectividad de un servicio (Vizcaíno, et. al.,2017).

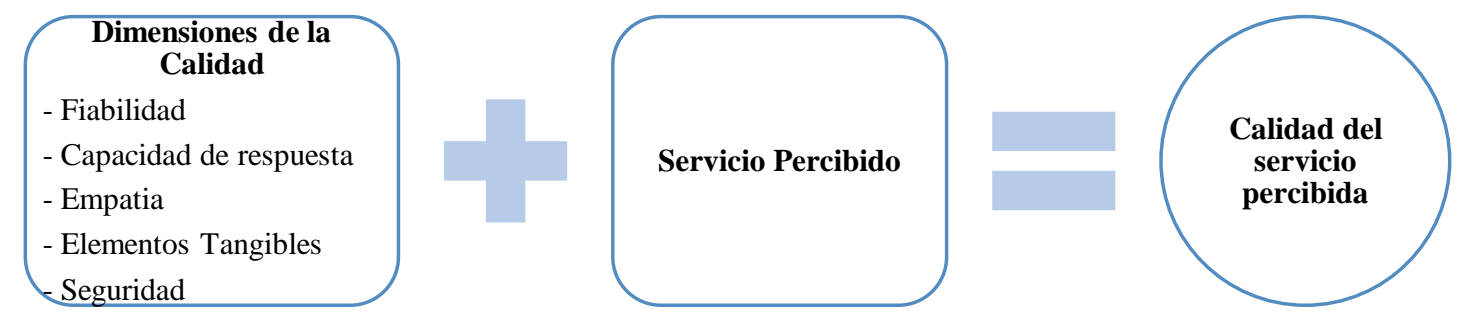

Figura 1. Modelo SERVPERF de calidad de servicio

Fuente: (Cronin y Taylor, 1992, pág. 128) 
El concepto de Calidad tiene un sin número de definiciones dependiendo de los múltiples enfoques que los investigadores desean resaltar en sus investigaciones, para lo cual, el trabajo presentado definirá la calidad desde la perspectiva del cliente, en este caso desde el punto de vista tanto el turista nacional como del extranjero, quienes son los que adquiere los servicios prestados en los establecimientos de alojamiento.

Con respecto a Varo, (1994) el concepto de calidad toma como base a la Real Academia Española en donde lo definen como "la propiedad o conjunto de propiedades inherentes a una cosa que permite apreciarla como igual, mejor o peor que las restantes de su misma especie" (pág.102). En definitiva, dicho concepto es extenso, en donde las organizaciones están en desarrollo mejorado sus productos y servicios con el fin de satisfacer los requerimientos y necesidades del cliente con el fin de ser competitivos.

Por tal motivo, algunos de los gurús más importantes de la historia que han estudiado el ámbito de la calidad a lo largo de sus carreras aportan diferentes significados de los cuales hay que mencionar en la recopilación de Hoyer y Hoyer, (2001) quien toma en consideración la explicación de la calidad en sus diversos aspectos y sus percepciones a su vez que exponen sus característica que se las presenta a continuación:

Tabla 1.

Teorías de la calidad

Autores

\section{Características}

Phil Crosby La calidad de un producto o servicio es equivalente a estar seguro de medir todas las características de un producto o servicio que satisfagan los criterios de especificación. "cero defectos"

Deming La calidad tiene que estar definida en términos de satisfacción del cliente que a su vez es multidimensional, es virtualmente imposible definir calidad de un producto o servicio en términos de una simple característica o agente.

Feigenbaum Los clientes tienen necesidades cambiantes, la calidad es dinámica, además se evalúa el nivel de calidad que esperan los clientes, así como lo que estarían dispuesto a pagar.

Ishikawa La definición de calidad es siempre cambiante, así mismo es equivalente a la satisfacción del cliente con enfoque a cada departamento en la organización que ofrece el producto o servicio, en donde el precio de un producto o servicio es una parte importante. No se puede definir calidad sin haber considerado antes el precio.

Juran La calidad consiste en aquellas características de producto que se basan en las necesidades del cliente y que por eso brindan satisfacción del producto.

Pirsig La calidad es como el arte moderno. No somos capaces de definir el buen arte moderno, pero lo reconocemos cuando lo vemos. 
Shewhart Existen dos características de calidad: subjetiva (lo que el cliente quiere) y objetiva (propiedades del producto o servicio). Se debe agregar que los estándares de calidad deben ser expresados en términos físicos y características cuantitativamente medibles de los productos por medio de la estadística.

Tagushi "La calidad es la pérdida que un producto causa a la sociedad después de haber sido entregado...algunas otras pérdidas son causadas por su función intrínseca."

Fuente: (Hoyer y Hoyer, 2001)

La calidad entendida como un proceso integrado compuesto por varios elementos Expectativas vs. Percepciones, según Parasuraman et al. $(1985,1988)$, citado por Velásquez (2011), parte de la conceptualización de Gronroos para desarrollar un instrumento que pueda medir la calidad de servicio percibida denominado SERVQUAL, el mismo que se basa en un extenso grupo de entrevistas, donde se identifican 10 determinantes de la calidad de servicio: 1) Elementos tangibles: apariencia de las instalaciones físicas, equipos, personal y materiales de comunicación; 2) Fiabilidad: habilidad para ejecutar el servicio prometido de forma fiable y cuidadosa; 3) Capacidad de respuesta: disposición para ayudar a los clientes, y para proveerlos de un servicio rápido; 4) Profesionalidad: posesión de las destrezas requeridas y conocimiento del proceso de prestación del servicio; 5) Cortesía: atención, consideración, respeto y amabilidad del personal de contacto; 6) Credibilidad: veracidad, creencia, y honestidad en el servicio que se provee; 7) Seguridad: inexistencia de peligros, riesgos o dudas; 8) Accesibilidad: lo accesible y fácil de contactar; 9) Comunicación: mantener a los clientes informados, utilizando un lenguaje que puedan entender, así como escucharlos y 10) Compresión del cliente: hacer el esfuerzo de conocer a los clientes y sus necesidades.

\section{Metodología}

La presente investigación es cuantitativa, tipo descriptiva según Horna (2012) porque recoge datos importantes sobre el potencial turístico de la práctica del rafting desarrollado en el cantón Tena, provincia de Napo; donde se expone y resume la información de manera secuencial, a fin de extraer elementos explicativos y concluyentes.

A partir de la escala y dimensiones basada en el modelo en el instrumento de evaluación SERVQUAL, se realizó el análisis de la calidad del servicio y satisfacción del cliente en la práctica de rafting en el cantón Tena, este método implicó inicialmente la realización de una encuesta a un grupo representativo de clientes (en este caso a turistas nacionales y extranjeros) con el fin de verificar si sus necesidades en la práctica de este deporte son atendidas satisfactoriamente por parte de las 22 agencias de viaje que operan y están debidamente capacitadas con los equipos y personal para guiar todo el recorrido de la actividad en el cantón Tena, catalogado por el Ministerio de Turismo como uno de los principales sitios donde se realiza actividades de aventura en la modalidad de Rafting, que según el Reglamento de Operación Turística de Aventura (2020) consiste en navegar ríos de aguas blancas en una balsa inflable tipo "raft", sin otro medio de propulsión y 
control de la embarcación que el generado por los mismos navegantes con el empleo de remos.

\section{Población de estudio}

La población objeto de estudio corresponde a los turistas nacionales y extranjeros como unidad de análisis que visitan el cantón Tena y practican la modalidad de rafting, la misma que se desconoce por tanto para calcular el tamaño de la muestra se aplica la fórmula para población infinita:

$$
n=\frac{z^{2} p * p}{d^{2}}
$$

\section{Procedimiento:}

$$
\begin{gathered}
\boldsymbol{n}=\frac{(\mathbf{1}, \mathbf{9 6})^{2}(0.5)(0,5)}{(0,05)^{2}} \\
n=384,16 \\
n=385 \text { encuestas }
\end{gathered}
$$

\begin{tabular}{|c|c|}
\hline Universo & $\begin{array}{l}\text { Turista que visitan el cantón Tena durante el mes de } \\
\text { febrero del } 2020\end{array}$ \\
\hline Ámbito geográfico & Cantón Tena \\
\hline Recogida de información & Cuestionario personal - encuesta \\
\hline Tamaño de la muestra & 385 \\
\hline Error muestral & 0.05 \\
\hline Probabilidad de éxito & 0.5 \\
\hline Probabilidad de fracaso & 0.5 \\
\hline Nivel de significación & $95 \%$ \\
\hline $\begin{array}{l}\text { Fecha del trabajo de } \\
\text { campo }\end{array}$ & Tercera y cuarta semana de febrero de 2020 \\
\hline
\end{tabular}

Tabla 2.

Ficha técnica del proceso metodológico

\section{Identificación de las variables:}

Variable Independiente: La calidad en el servicio

Variable Dependiente: Satisfacción del cliente

\section{Hipótesis de estudio:}

El Modelo Servqual, permite analizar las expectativas y percepciones de los clientes sobre un servicio, a través de las cinco dimensiones de la calidad, que se pueden relacionar con la satisfacción del cliente. Por tanto, se plantea la siguiente hipótesis:

H1. Existe relación directa entre la calidad del servicio y la satisfacción del cliente 
H2. En base a las dimensiones afectan directamente a la satisfacción del cliente

\section{Modelo de brechas}

- $\quad$ Brecha 1: Diferencia entre las expectativas de los clientes y las percepciones de los directivos de la empresa. Si los directivos de la organización no comprenden las necesidades de los clientes, difícilmente podrán impulsar y desarrollar acciones para lograr la satisfacción de esas necesidades y expectativas

- Brecha 2: Diferencia entre las percepciones de los directivos y las especificaciones de las normas de calidad. El factor que debe estar presente para evitar esta brecha es la traducción de las expectativas, conocida a especificaciones de las normas de la calidad del servicio.

- $\quad$ Brecha 3: Discrepancia entre las especificaciones de la calidad del servicio y la prestación del servicio. La calidad del servicio no será posible si las normas y procedimientos no se cumplen.

- Brecha 4: Discrepancia entre la prestación del servicio y la comunicación externa. Para el Servqual, uno de los factores claves en la formación de las expectativas, es la comunicación externa de la organización proveedora. Las promesas que la empresa hace, y la publicidad que realiza afectara a las expectativas.

- Brecha 5: Es la diferencia entre las expectativas de los clientes frente a las percepciones de ellos.

- En esta investigación se analizó solo la brecha cinco, que permitió identificar las causas que originan el problema, haciendo un comparativo entre las expectativas y las percepciones de los clientes.

\section{Modelos estadísticos utilizados}

En la presente investigación se utilizó un método de comprobación de hipótesis que es la correlación lineal y análisis de la varianza con un solo factor

\section{El coeficiente de correlación}

El coeficiente de correlación lineal es el cociente entre el error estándar estimado y el producto de las desviaciones típicas de ambas variables

\begin{tabular}{lcc}
\hline Error estándar estimado & $\begin{array}{c}\text { Varianza de y } \\
\text { (independiente) }\end{array}$ & Coeficiente de correlación \\
\hline Se & $S_{y}^{2}=\frac{\sum y^{2}}{N}-\bar{y}^{2}$ & $r^{2}=1-\frac{S e^{2}}{S_{y}^{2}}$ \\
$=\sqrt{\frac{\sum y^{2}-\boldsymbol{a} \sum \boldsymbol{y}-\boldsymbol{b} \sum \boldsymbol{x} * \boldsymbol{y}}{\boldsymbol{n}-\mathbf{2}}}$ & &
\end{tabular}




\section{Análisis de la varianza con un solo factor ANOVA Unifactorial}

Es una técnica estadística que señala si dos variables (una independiente y otra dependiente) están relacionadas en base a si las medias de la variable dependiente son diferentes en las categorías o grupos de la variable independiente. Es síntesis, señala si las medias entre dos o más grupos son similares o diferentes.

\section{Resultados}

El potencial turístico de la práctica de rafting que se desarrolla en el cantón Tena, provincia de Napo, se encuentra en la parroquia Talag, donde está el río Jatunyaku, que es considerado por los expertos como el mejor río para hacer rafting en Ecuador, por poseer una corriente perfecta que permite la práctica este deporte a cualquier nivel. También, es un área de increíble de riqueza natural (flora endémica) y de una biodiversidad única en el mundo.

El recorrido abarca aproximadamente los $35 \mathrm{~km}$ y atraviesa por comunidades asentadas a la orilla del río Jatunyaku, se identificaron 3 paradas estratégicas Tena - Serena (distancia: $18,5 \mathrm{~km}$ ), Serena - Waysayaku (distancia: 7,6 Km) y Cando - Puerto Napo (distancia: $26 \mathrm{Km}$ ), tal como se visualiza a continuación en la Figura 2:

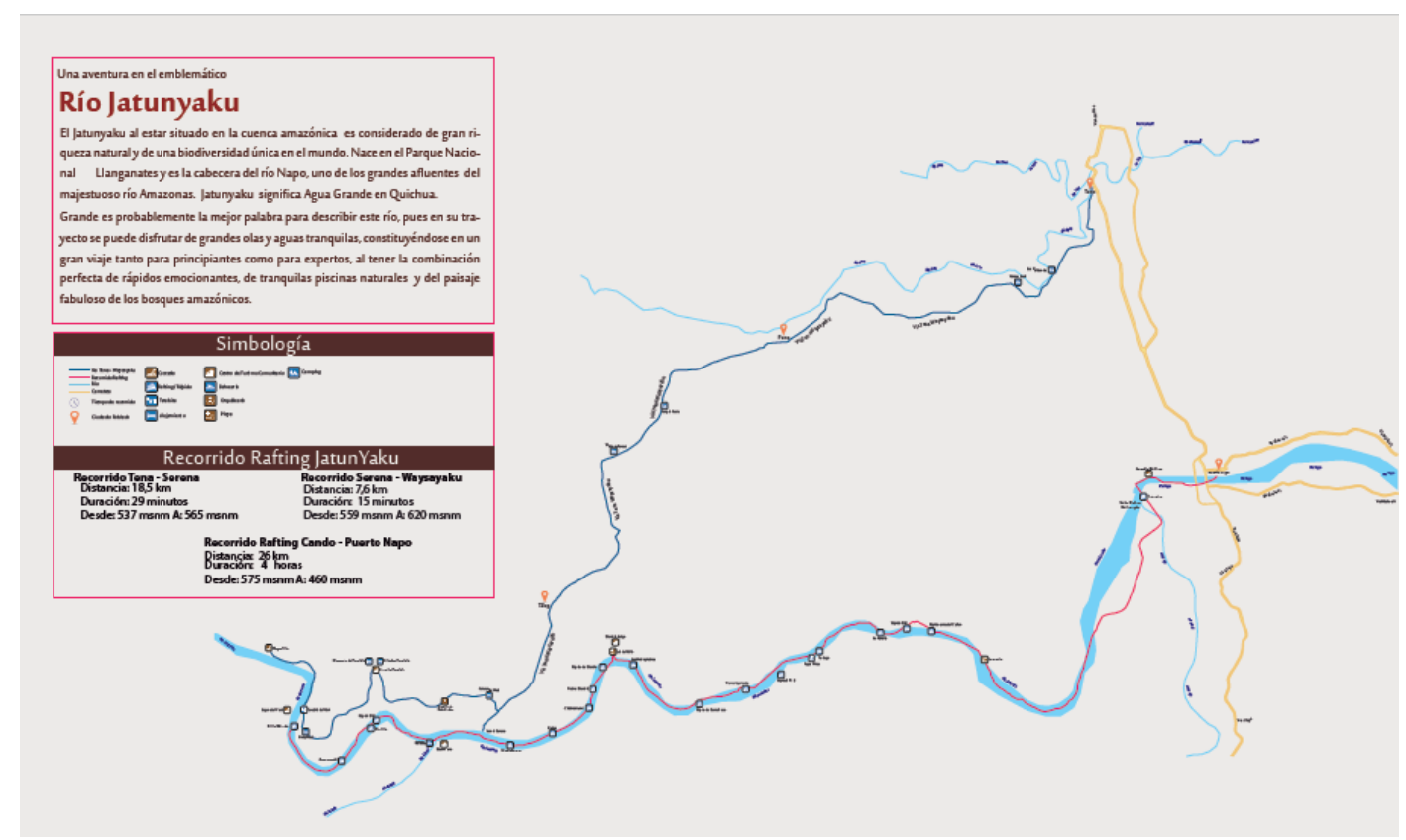

Figura 2. Recorrido Rafting río Jatunyaku

Fuente: (GAD Municipal de Tena, 2019)

Tabla 3.

Georreferenciación Río Jatunyaku

\begin{tabular}{cll}
\hline Nro & \multicolumn{1}{c}{ LUGAR } & \multicolumn{1}{c}{ GEOREFERENCIA } \\
\hline 1 & Cabañas Pimpilala & $-1.0762964,-77.937195$ \\
2 & Cascadia napo & $-1.0444496,-77.804535$ \\
3 & Cascadita Río Napo & $-1.04445,-77.80453$ \\
\hline
\end{tabular}




\begin{tabular}{|c|c|c|}
\hline 4 & El Encanto de Pimpilala & $-1.07627,-77.93723$ \\
\hline 5 & Fin de Recorrido Pto. Napo & $-1.04347,-77.79328$ \\
\hline 6 & Isla del gallo Shandia & $-1.07301,-77.89578$ \\
\hline 7 & Jatun Wasi - Alojamiento & $-1.08081,-77.91786$ \\
\hline 8 & La cuevita & $-1.07445,-77.83295$ \\
\hline 9 & Laguna del Yani & $-1.0832046,-77.949776$ \\
\hline 10 & Orquideario Ruth Garden & $-1.08185,-77.92547$ \\
\hline 11 & Piedra antes de Shandia & $-1.07972,-77.90006$ \\
\hline 12 & Puente Serena & $-1.08677,-77.91916$ \\
\hline 13 & Rápido Amaroncachi & $-1.0919025,-77.943085$ \\
\hline 14 & Rápido antes del Cañon & $-1.06973,-77.84199$ \\
\hline 15 & Rápido Chichicirumi & $-1.0829757,-77.900795$ \\
\hline 16 & Rápido Chichicirumi & $-1.08297,-77.90079$ \\
\hline 17 & Rápido de Santa Rosa & $-1.08224,-77.88187$ \\
\hline 18 & Rápido de Shandia & $-1.07591,-77.89832$ \\
\hline 19 & Rápido Hatles & $-1.08724,-77.90699$ \\
\hline 20 & Rápido Jardín de piedras & $-1.07548,-77.89314$ \\
\hline 21 & Rápido La Natalia & $-1.06994,-77.85077$ \\
\hline 22 & Rápido Papas Fritas & $-1.07428,-77.86257$ \\
\hline 23 & Rápido Pierna izquierda & $-1.07969,-77.87391$ \\
\hline 24 & Rápido Pimpilala & $-1.0863559,-77.93811$ \\
\hline 25 & Rápido S/N & $-1.08503,-77.93715$ \\
\hline 26 & Rápido S/N 3 & $-1.06926,-77.8463$ \\
\hline 27 & Rápido S/N 2 & $-1.0771,-77.86778$ \\
\hline 28 & Rápido Serena & $-1.08927,-77.91419$ \\
\hline 29 & Rápido Tortuga & $-1.07309,-77.86118$ \\
\hline 30 & Rápido Yutzupino & $-1.04722,-77.8069$ \\
\hline 31 & Río Illoculín & $-1.08859,-77.92722$ \\
\hline 32 & Salida El Cando & $-1.08655,-77.9515$ \\
\hline 33 & Unión Río Jatunyaku y Río Anzu & $-1.05154,-77.80268$ \\
\hline 34 & Waysa Yaku & $-1.07377,-77.95503$ \\
\hline
\end{tabular}

\section{Tabla 4.}

Ficha información técnica

\begin{tabular}{ll}
\hline Ecosistema: & $\begin{array}{l}\text { Sistema de bosque primario y secundario, montañoso } \\
\text { irregular. }\end{array}$ \\
Clima: & $\begin{array}{l}\text { Cálido - húmedo con temperaturas de } 23{ }^{\circ} \mathrm{C} \\
\text { Desde los meses de octubre a marzo son los meses con } \\
\text { niveles de agua óptimos para el rafting y se los puede hacer } \\
\text { en la mayoría de los ríos. }\end{array}$ \\
Zona cartográfica: & Latitud: -1.06667 \\
& Longitud: -77.8 \\
Grado de dificultad: & Medio \\
Distancia (m): & $35 \mathrm{~km}$ \\
Altitud (msnm): & $480 \mathrm{msnm}$ \\
Tiempo (horas): & 6 horas \\
Tipo de camino: & Aguas rápidas \\
\hline
\end{tabular}


Para nivel de satisfacción de los turistas nacionales y extranjeros con respecto a la calidad de los servicios turísticos del rafting, una vez recolectada la información (encuesta Servqual aplicada a los clientes) en las AAVV del cantón Tena, sobre la oferta del servicio de rafting que están dentro del campo de estudio y después de plantear los métodos estadísticos adecuados (coeficiente correlación y Análisis de la varianza ANOVA unifactorial) se presenta los siguientes resultados, para resolver las hipótesis del presente estudio.

\section{Tabla 5.}

Dimensión de Tangibilidad

\begin{tabular}{clcc}
\hline $\mathbf{N}$ & Variable & Calificación & $\begin{array}{c}\text { Nivel de } \\
\text { Satisfacción }\end{array}$ \\
\hline $\mathbf{1}$ & $\begin{array}{l}\text { La agencia de viajes cuenta con equipos de apariencia } \\
\text { moderna. }\end{array}$ & 4,1 & $81,3 \%$ \\
\hline $\mathbf{2}$ & $\begin{array}{l}\text { El aspecto interno y externo de la agencia de viajes es } \\
\text { visualmente atractivo. }\end{array}$ & 3,8 & $79,6 \%$ \\
\hline $\mathbf{3}$ & $\begin{array}{l}\text { Existe comodidad, limpieza y los espacios son } \\
\text { confortables. }\end{array}$ & 4,2 & $83,2 \%$ \\
\hline $\mathbf{4}$ & $\begin{array}{l}\text { Los elementos materiales POP (trípticos, contratos y } \\
\text { similares) son visualmente atractivos. }\end{array}$ & 4,0 & $80,5 \%$ \\
\hline
\end{tabular}

Fuente: (Encuestas, 2020)

En la dimensión de Tangibilidad; el cliente considera las expectativas que tiene por las instalaciones, siendo mayor a lo que realmente percibe, en este punto la apariencia del establecimiento es muy buena, por otro lado, considera que el establecimiento interno y externo del establecimiento no tienen un buen porcentaje $(79,6 \%)$ en el cual se sustenta con los aspectos visuales e informativos que disponen las agencias de viaje, el mismo que no es el esperado por el cliente.

\section{Tabla 6}

Dimensión de fiabilidad

\begin{tabular}{clcc}
\hline $\mathbf{N}$ & \multicolumn{1}{c}{ Variable } & Calificación & \multicolumn{1}{c}{$\begin{array}{c}\text { Nivel de } \\
\text { Satisfacción }\end{array}$} \\
\hline $\mathbf{1}$ & $\begin{array}{l}\text { El personal de la AAVV, cumple con lo prometido } \\
\text { en el tiempo pactado }\end{array}$ & 4,0 & $80,5 \%$ \\
\hline $\mathbf{2}$ & $\begin{array}{l}\text { El personal de AAVV resuelve problemas de los } \\
\text { clientes y muestra interés por solucionarlos }\end{array}$ & 4,2 & $83,2 \%$ \\
\hline $\mathbf{7}$ & La AAVV cumple con los horarios establecidos & 4,1 & $81,3 \%$ \\
\hline $\mathbf{4}$ & $\begin{array}{l}\text { La AAVV concluye el servicio en el tiempo } \\
\text { prometido }\end{array}$ & 4,1 & $81,3 \%$ \\
\hline $\mathbf{5}$ & $\begin{array}{l}\text { La AAVV es eficiente y mantienen registros exentos } \\
\text { de errores en el servicio }\end{array}$ & 4,0 & $80,5 \%$ \\
\hline
\end{tabular}

Fuente: (Encuestas, 2020)

En la dimensión de fiabilidad, los encuestados (clientes) manifiestan como alto nivel de satisfacción que el personal de la AAVV resuelve rápidamente sus problemas, existen además clientes que consideran el tiempo de espera al momento de obtener el servicio como muy bueno o eficiente considerando que la eficiencia del servicio no es lo esperado. 


\section{Tabla 7}

Dimensión de la capacidad de respuesta

\begin{tabular}{rlrr}
\hline $\mathbf{N}$ & \multicolumn{1}{c}{ Variable } & Calificación & \multicolumn{1}{c}{$\begin{array}{c}\text { Nivel de } \\
\text { Satisfacción }\end{array}$} \\
\hline $\mathbf{1}$ & La atención brindada por la AAVV es rápida & 4,1 & $81,3 \%$ \\
\hline $\mathbf{2}$ & Disponibilidad de asistir y asesorar a sus clientes & 4,1 & $81,3 \%$ \\
\hline $\mathbf{3}$ & Existe una comunicación del cliente-personal & 4,0 & $80,5 \%$ \\
\hline $\mathbf{4}$ & $\begin{array}{l}\text { El personal de la AAVV siempre está dispuestos a } \\
\text { contestar todas sus interrogantes }\end{array}$ & 4,0 & $81,3 \%$ \\
\hline
\end{tabular}

Fuente: (Encuestas, 2020)

En la dimensión de la capacidad de respuesta, se considera que el personal de la AAVV siempre esta dispuesto con el público, por lo que recibió una percepción muy buena, sobre la variable de disponibilidad de asistir y asesorar al cliente en cual se obtuvo un promedio de $81.3 \%$, con lo que se concluye que la prestación de servicio es disponible y rápida en las agencias de viaje del cantón Tena.

\section{Tabla 8}

Dimensión de confianza

\begin{tabular}{|c|c|c|c|}
\hline $\mathbf{N}$ & Variable & Calificación & $\begin{array}{c}\text { Nivel de } \\
\text { Satisfacción }\end{array}$ \\
\hline 1 & $\begin{array}{l}\text { Existe seguridad en las instalaciones y equipos de } \\
\text { la AAVV }\end{array}$ & 4,1 & $81,3 \%$ \\
\hline 2 & Muestra capacidad de organización del servicio & 4,2 & $83,2 \%$ \\
\hline 3 & $\begin{array}{l}\text { La AAVV trasmite confianza al ofrecer el } \\
\text { servicio }\end{array}$ & 4,1 & $82,0 \%$ \\
\hline 4 & $\begin{array}{l}\text { La AAVV tiene un grado de conocimiento al } \\
\text { resolver problemas }\end{array}$ & 4,0 & $80,5 \%$ \\
\hline 5 & Amabilidad y cortesía de la AAVV & 4,0 & $80,5 \%$ \\
\hline
\end{tabular}

Fuente: (Encuestas, 2020)

En la dimensión de confianza; se observa que la confianza del servicio fue calificada en promedio, donde se considera que los clientes recibieron servicios confiables y de calidad, con el respecto a la variable de seguridad dentro de las instalaciones y equipos se refiere al nivel de confianza que brinda el personal en donde se obtuvo una calificación de 4,10, que es considerado como muy bueno.

\section{Tabla 9}

\section{Dimensión de empatía}

\begin{tabular}{llcc}
\hline $\mathbf{N}$ & \multicolumn{1}{c}{ Variable } & Calificación & $\begin{array}{c}\text { Nivel de } \\
\text { Satisfacción }\end{array}$ \\
\hline $\mathbf{1}$ & $\begin{array}{l}\text { La AAVV tiene horarios de atención } \\
\text { convenientes }\end{array}$ & 4,2 & $83,2 \%$ \\
\hline $\mathbf{2}$ & Grado de comprensión del personal de la AAVV & 4,0 & $80,5 \%$ \\
\hline
\end{tabular}




\begin{tabular}{llcc}
3 & $\begin{array}{l}\text { Preocupación y capacidad para resolver } \\
\text { inquietudes. }\end{array}$ & 4,0 & $80,5 \%$ \\
$\mathbf{4}$ & $\begin{array}{l}\text { La relación con los clientes para entender su } \\
\text { requerimiento }\end{array}$ & 4,1 & $82,0 \%$ \\
\hline
\end{tabular}

Fuente: (Encuestas, 2020)

En la dimensión de empatía; el horario de atención para el uso del servicio es considerado como fundamental por los clientes obteniendo una calificación promedio de 4,16 y con un porcentaje del nivel de satisfacción de $83,25 \%$, se concluye así que las agencias de viaje están dispuestas a brindar sus servicios en horarios adecuados para el desarrollo de esta práctica.

\section{Índice de Calidad de Servicio}

Con el resultado de las puntuaciones de la encuesta aplicada, se procedió a realizar un análisis cuantitativo de la percepción donde se obtuvo una sumatoria y el cual está divido por la cantidad del tamaño de la muestra. Para cada dimensión de la calidad de servicio se tiene un promedio que se puede visualizar en la siguiente gráfica:

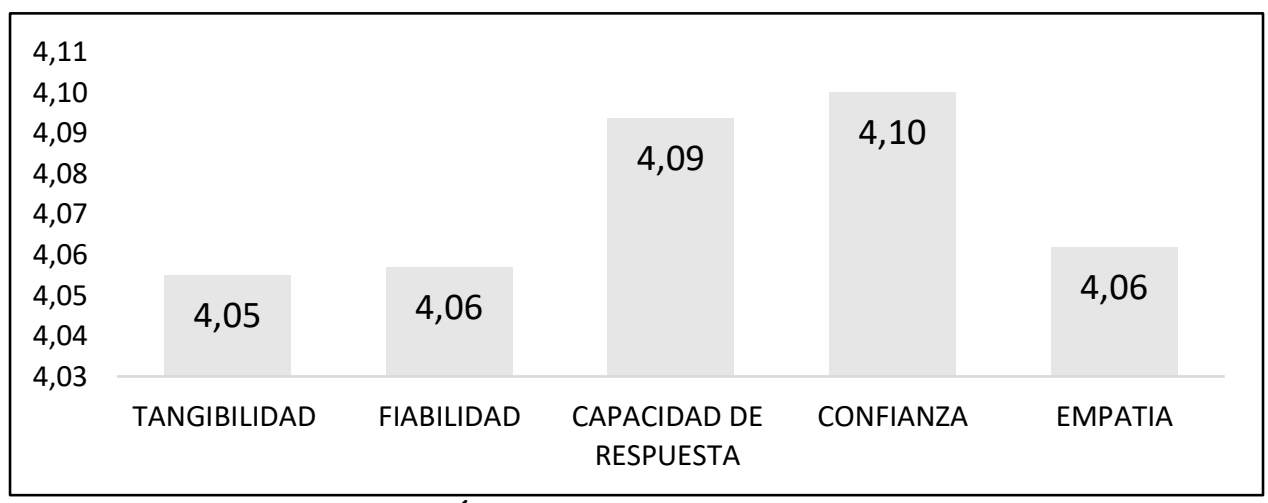

Grafica 1. Índice de Calidad de Servicio

Fuente: (Encuestas, 2020)

La dimensión con menor promedio de percepción es tangibilidad, después está la fiabilidad y empatía. El cálculo del promedio general de las 5 dimensiones evaluadas es de 4.06 lo que representa un $81.44 \%$ de los clientes que perciben un buen servicio de calidad según el grado de porcentaje $0-100 \%$ de la escala de Likert representado en la siguiente tabla:

\section{Tabla 10}

Nivel y puntos de Escala de Likert

\begin{tabular}{c|c|l}
\hline $\begin{array}{c}\text { NIVEL Y PUNTOS DE } \\
\text { ESCALA DE LIKERT }\end{array}$ & $\begin{array}{c}\text { RANGO PORCETAJE } \\
\text { SATISFACCIÓN CLIENTE }\end{array}$ & \multicolumn{1}{|c}{$\begin{array}{c}\text { CALIFICACIÓN } \\
\text { DEL SERVICIO }\end{array}$} \\
\hline 1 & $0 \%-20 \%$ & Muy mala calidad \\
\hline 2 & $20 \%-40 \%$ & Baja calidad \\
\hline 3 & $40 \%-60 \%$ & Normal \\
\hline 4 & $60 \%-80 \%$ & Buena calidad \\
\hline 5 & $\mathbf{8 0 \%}-\mathbf{1 0 0 \%}$ & Muy buena calidad \\
\hline
\end{tabular}

Cabe indicar que, en la dimensión de tangibilidad, se determina que el mayor error al brindar el servicio se encuentra en el "aspecto interno y externo de la agencia de viajes es 
visualmente atractivo las instalaciones físicas del establecimiento" por lo que esta variable recibe un promedio inferior al resto y debe ser mejorada.

\section{Satisfacción del cliente: Análisis GAP}

Al realizar el análisis del comportamiento del promedio de cada una de las calificaciones de las percepciones con las expectativas, observamos la brecha existente, es decir que la diferencia percibida entre lo esperado del servicio y lo que recibe el cliente, según lo muestra la siguiente tabla:

\section{Tabla 11}

Brechas de las dimensiones de la calidad

\begin{tabular}{lccc}
\hline DIMENSIÓNES & PERCEPCIÓNES & EXPECTATIVAS & BRECHA \\
\hline Tangibilidad & 4,05 & 4,11 & $-0,06$ \\
\hline Fiabilidad & 4,06 & 4,11 & $-0,06$ \\
\hline Capacidad de respuesta & 4,09 & 4,11 & $-0,02$ \\
\hline Confianza & 4,10 & 4,11 & $-0,01$ \\
\hline Empatía & 4,06 & 4,11 & $-0,05$
\end{tabular}

Fuente: (Encuestas, 2020)

Todas las dimensiones utilizadas presentan brechas negativas, es decir se detecta fallas en la calidad, esta información brinda la oportunidad de mejorar en cada una de las dimensiones, en los puntos más débiles particularmente del servicio de rafting que brindan las AAVV, sobre las dimensiones de Tangibilidad, fiabilidad y empatía.

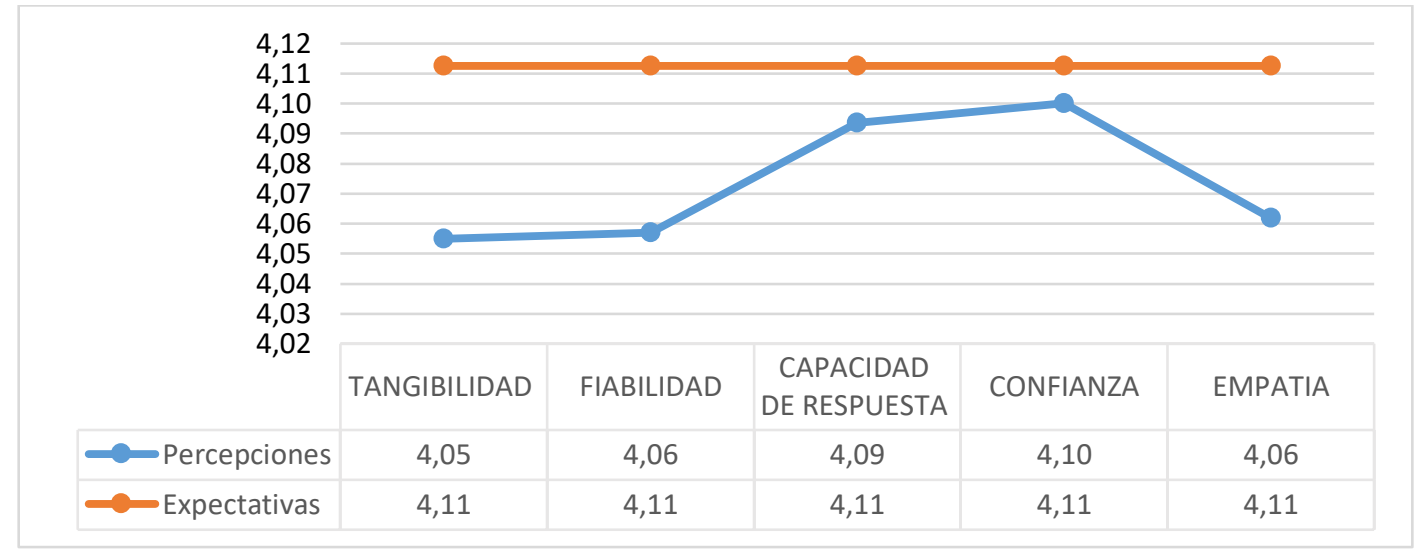

Gráfico 2. Brechas de las dimensiones de la calidad.

Fuente: (Encuestas, 2020)

\section{Comprobación de hipótesis}

Los resultados de los análisis estadísticos muestran las pruebas realizadas con cada una de las variables explicativas utilizando el coeficiente de correlación y el ANOVA unifactorial, después se presentan los resultados derivados con el modelo de coeficiente de correlación multivariado, esto tomando la distribución de cada una de las hipótesis planteadas. 


\section{H1. Relación directa entre la calidad del servicio y la satisfacción del cliente}

A continuación, se observa los estadísticos de las encuestas realizadas a los clientes del sector de alojamiento de la ciudad de Puyo, para establecer la correlación lineal.

\section{Tabla 12}

Correlación Lineal

\section{EXPECTATIVAS PROMEDIO}

\begin{tabular}{llll}
\hline EXPECTATIVAS & $\begin{array}{l}\text { Correlación de } \\
\text { Pearson }\end{array}$ & 1 & $.828^{* *}$ \\
\cline { 2 - 3 } & Sig. (unilateral) & .000
\end{tabular}

$\star \star$. La correlación es significativa en el nivel 0,01 (unilateral).

Fuente: (Encuestas SPSS)

Con esto se puede establecer que la satisfacción del cliente entendido como "una sensación de placer o de decepción que resulta de comparar la experiencia del producto y/o servicios esperados, con las expectativas de beneficios previas. Si los resultados son inferiores a las expectativas el cliente queda insatisfecho.

Si los resultados son están a la altura de las expectativas, el cliente queda satisfecho. Si los resultados superan las expectativas, el cliente queda muy satisfecho o encantado".

\section{Tabla 13}

ANOVA unifactorial

\begin{tabular}{lcrrrr}
\hline Modelo & $\begin{array}{c}\text { Suma de } \\
\text { cuadrados }\end{array}$ & gl & \multicolumn{1}{c}{$\begin{array}{c}\text { Media } \\
\text { cuadrática }\end{array}$} & F & Sig. \\
\hline Regresión & 2514949.790 & 5 & 502989.958 & 174.044 & $.000^{\mathrm{b}}$ \\
\hline Residuo & 1086647.069 & 376 & 2890.019 & & \\
\hline Total & 3601596.859 & 381 & & & \\
\hline
\end{tabular}

\section{ANOVA $^{\mathrm{a}}$}
a. Variable dependiente: EXPECTATIVAS
b. Predictores: (Constante), EMPATIA, TANGIBILIDAD, FIABILIDAD,
CAPACIDAD_DE_RESPUESTA, CONFIANZA

Confirmando los datos de la correlación lineal de Pearson el Análisis de Variancia Unificado esta demuestra una significancia de 0,000 que menciona que son predictores: Empatía, Tangibilidad, Fiabilidad, Capacidad de respuesta, Confianza.

En base a las dimensiones de la calidad del servicio afectan directamente a la satisfacción del cliente 
En la tabla de coeficiente de correlación múltiple se observa los estadísticos de las encuestas realizadas a los clientes, estos datos muestran diferentes puntos de análisis entre cada una de las dimensiones del SERVQUAL y la satisfacción del cliente.

Existe una significancia entre la dimensión de tangibilidad y satisfacción de 0,270 es decir no tienen relación directa, entre fiabilidad y satisfacción de un 0,025 que menciona que son relacionantes entre las dos variables.

Mientras que para capacidad de respuesta y el cliente se observa un 0,612, tomando los datos de confianza y satisfacción los cuales son 0,000 se puede indicar que son relacionantes y pro ultimo la empatía y la satisfacción del cliente que son 0,000 que son correlacionantes.

\section{Tabla 14}

\section{Coeficiente de correlación múltiple}

\begin{tabular}{|c|c|c|c|c|c|}
\hline \multirow{2}{*}{ Modelo } & \multicolumn{2}{|c|}{$\begin{array}{l}\text { Coeficientes no } \\
\text { estandarizados }\end{array}$} & \multirow{2}{*}{$\begin{array}{c}\begin{array}{c}\text { Coeficientes } \\
\text { estandarizados }\end{array} \\
\text { Beta }\end{array}$} & \multirow{2}{*}{$\mathbf{t}$} & \multirow{2}{*}{ Sig. } \\
\hline & B & $\begin{array}{l}\text { Error } \\
\text { estándar }\end{array}$ & & & \\
\hline TANGIBILIDAD & .072 & .066 & .057 & 1.105 & .270 \\
\hline FIABILIDAD & .171 & .076 & .144 & 2.245 & .025 \\
\hline CAPACIDAD_DE_RESPUESTA & .042 & .083 & .035 & .507 & .612 \\
\hline CONFIANZA & .312 & .088 & .264 & 3.535 & .000 \\
\hline EMPATIA & .452 & .089 & .380 & 5.102 & .000 \\
\hline
\end{tabular}

a. Variable dependiente: EXPECTATIVAS

Fuente: (Encuestas SPSS)

En esta investigación la satisfacción y calidad se relacionan directamente lo cual es positivo, a primera vista, ya que existen muchos factores que deben ser considerados entre ambos aspectos, lo que resulta muy complejidad para el cliente, lo importante del estudio es enfocarse en aquellos donde la mejora de estos factores puede establecer la percepción de atributos positivos para el cliente.

\section{Conclusiones}

- La actividad de aventura, en la modalidad de Rafting, tiene potencial turístico en el rio Jatunyaku, ya que cuente con 3 paradas estratégicas Tena - Serena (distancia: 18,5 km), Serena - Waysayaku (distancia: 7,6 Km) y Cando - Puerto Napo (distancia: $26 \mathrm{Km}$ ), 19 rápidos y una zona de buceo, que lo hacen atractivo a este deporte que provoca sensación de liberta y adrenalina para el turista extranjero y que puede ser aprovechado como un producto turístico del destino Tena.

- Las brechas más altas con la percepción del cliente y su expectativa son tangibilidad $(-0,06)$, fiabilidad $(-0,06)$ y empatía $(-0,05)$, por tanto, constituyen 
puntos primordiales donde es necesario plantear estrategias que mejoren la percepción sobre la calidad del servicio.

- En el análisis de las hipótesis, existe un coeficiente de correlación entre la calidad del servicio y la satisfacción del cliente de un 0,828 y una significativa de 0,01 estableciendo una relación directa entre la calidad de servicio y la satisfacción del cliente.

\section{Referencias bibliográficas}

Cronin Jr, J. J., \& Taylor, S. A. (1992). Measuring service quality: a reexamination and extension. Journal of marketing, 56(3), 55-68.

Camara, C. J., \& Ángeles, F. (2014). Metodología para la identificación, clasificación y evaluación de los recursos territoriales turísticos del centro de ciudad de Fortde-France. Santiago de Cuba-Cuba : Facultad de Construcciones. Universidad de Oriente. .

Daza, J. (2013). Análisis de la medición de calidad en los servicios hoteleros. Criterio Libre(19), 263-280.

García Morales, G. (2012). EVALUACIÓN DEL POTENCIAL TURÍSTICO EN LA PLAYA NORTE DE EL MOGOTE, BAHÍA DE LA PAZ, B.C.S., MÉXICO. LA PAZ, B.C.S.: INSTITUTO POLITECNICO NACIONAL.

Gil Saura, I. S.-G. (2005). Encuentro de servicio, valor percibido y satisfacción del cliente en la relación entre empresas. Cuadernos de Estudios Empresariales, 47-72.

Horna, A. A. (2012). 7 pasos para una tesis exitosa. En A. A. Horna. Lima: Universidad de San Martín de Porres.

Khattab, A., \& Aldehayyat. (2011). Perceptions of Service Quality in Jordanian Hotels. International Journal of Business and Management(07), 226-236. Obtenido de http://search.proquest.com/openview/6841122ccd6d421da1c1576a81f1ab09/1?p q-origsite $=$ gscholar

Mikery Gutiérrez, M. J. (2014). Potencial agroturístico de Tepexilotla, Veracreuz, México. Veracruz-México: Colegio de Potsgraduados-Campus Veracruz.

Ministerio de Turismo. (2020). Reglamento de Operación Turística de aventura. QuitoEcuador: Recuperado el 29 de febrero del 2021, de https://www.turismo.gob.ec/wpcontent/uploads/downloads/2020/08/Reglamento-de-Operacio\%CC\%81nTuri\%CC\%81stica-de-Aventura-2020.pdf.

Saghier, E. (2013). Managing Service Quality: Dimensions of service quality: a study in Egypt. Journal of Strategic and International Studies(3), 90-99. Recuperado el 28 


de Febrero de 2021, de http://www.isisworld.org/wp-
content/uploads/JSIS_May2014.pdf\#page $=90$

Thompson, I. (2005). La Satisfacción del Cliente. Boliva- La Paz: Rev Med.

Varo, J. (1994). Gestión estratégica de la calidad en los servicios sanitarios: un modelo de gestión hospitalaria. Díaz de Santos.

Velásquez, D. (2011). Medición de la satisfacción de usuarios de la Universidad del Valle.Cali: Univalle.

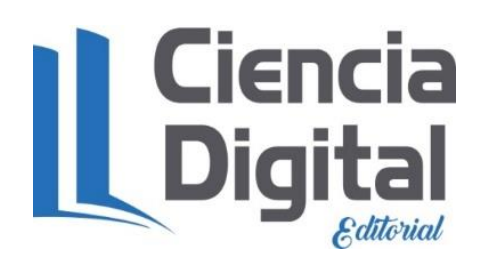




\section{PARA CITAR EL ARTÍCULO INDEXADO.}

Toalombo Díaz, Álvaro S., Cevallos Punguil, T. C., Rivadeneyra Olalla, G. P., \& Aguilar Soria, E. A. (2021). Análisis de la calidad del servicio y la satisfacción del cliente en la práctica de rafting en el cantón Tena. ConcienciaDigital, 4(2.2), 153-171. https://doi.org/10.33262/concienciadigital.v4i2.2.1784

\section{LCiencia}

El artículo que se publica es de exclusiva responsabilidad de los autores y no necesariamente reflejan el pensamiento de la Revista Conciencia Digital.

El artículo queda en propiedad de la revista y, por tanto, su publicación parcial y/o total en otro medio tiene que ser autorizado por el director de la Revista Conciencia Digital.

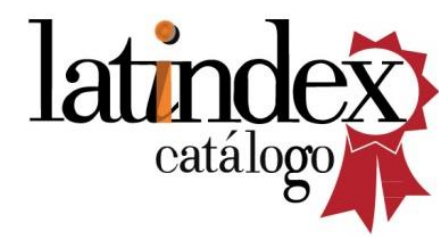

